\title{
Combined occlusion of the central retinal artery and central retinal vein following blunt ocular trauma: a case report
}

\author{
M J NOBLE AND E V ALVAREZ \\ From the Tennent Institute of Ophthalmology, University of Glasgow, Western Infirmary, Glasgow
}

SUMmaRY A healthy young woman suffered complete loss of the vision of one eye following a blunt ocular injury. She sustained a combined occlusion of the central retinal artery and central retinal vein of the affected eye. Initially few retinal haemorrhages were present, but they increased considerably in number and size during the day following injury.

Occlusion of the central retinal artery, the central retinal vein, or branches of these vessels, are well recognised events. Combined simultaneous occlusion of the central retinal artery and central retinal vein occurs very rarely, and has generally been observed in patients with systemic disorders such as leukaemia.' We report a case of a young woman who suffered a combined retinal vascular occlusion following blunt trauma to one eye. To our knowledge this is the first time that such a sequence of events has been recorded.

\section{Case report}

A 28-year-old woman was jabbed in the left eye by a finger during an altercation. She experienced immediate and total loss of the vision of this eye. She was in good health, and she had no past history of ocular disease.

One hour after this injury she attended the casualty department of the Glasgow Eye Infirmary, where her right visual acuity was found to be $6 / 6$ unaided, and the left was no perception of light. No abnormality of the right eye was detected. The left eyelids were mildly bruised. There was a linear conjunctival laceration $1.5 \mathrm{~cm}$ in length, above and parallel to the limbus. No perforation of the cornea or sclera was found. The ocular media were clear. The left pupil was round, and reacted consensually but not directly to light. The intraocular pressure of each eye was $12 \mathrm{mmHg}$. There was no proptosis of the left eye.

Correspondence to Dr M J Noble, Tennent Institute of Ophthalmology, University of Glasgow, Western Infirmary, Glasgow G11 6NT.
The left optic disc was abnormally pale, and the retinal arterioles were extremely narrow. There was a cherry-red spot at the macula. The retinal veins were grossly engorged, with segmentation of the vascular column. A few small perivenous haemorrhages were seen.

Initial treatment consisted of intravenous acetazolamide $500 \mathrm{mg}$, and carbon dioxide rebreathing, on the assumption that the blunt injury might have induced central retinal arterial spasm.

After a further 30 minutes the left vision remained no perception of light. The left ocular pressure had fallen to $6 \mathrm{mmHg}$. The retinal arterioles remained very narrow. The retinal haemorrhages had increased considerably in both number and size.

During the following day there was a further increase in the number of retinal haemorrhages, with the formation of some preretinal haemorrhages. The left optic disc became grossly swollen (Fig. 1).

Fluorescein angiography, performed on the day after injury, confirmed the absence of perfusion of the left central retinal artery and vein. Choroidal perfusion was maintained. Later frames of the angiogram showed slight filling of the proximal retinal arterioles (Fig. 2).

The patient subsequently failed to regain any vision in the left eye.

\section{Discussion}

Simultaneous occlusion of both the central retinal artery (CRAO) and the central retinal vein (CRVO) is a rare event. Occasional reports show it has been observed in association with disorders such 


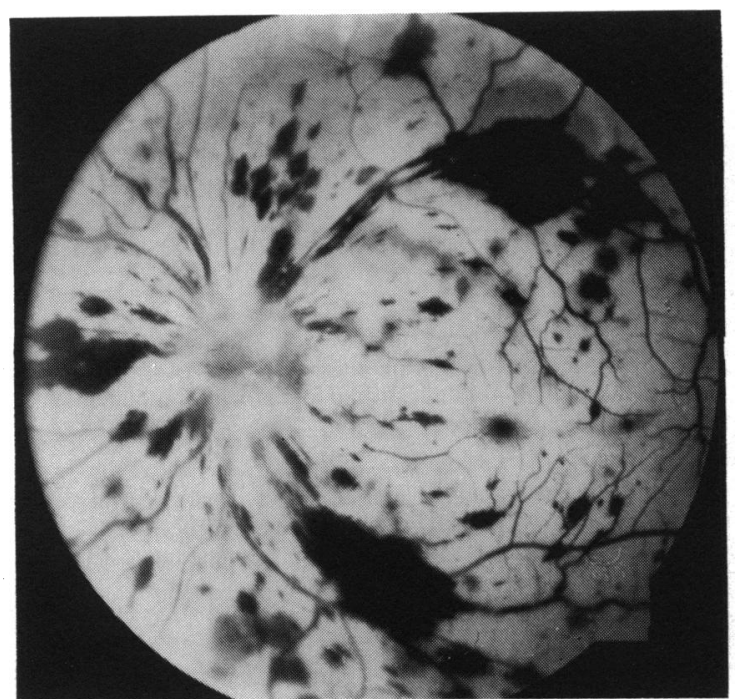

Fig. 1 Photograph of the posterior pole of the left eye one day after injury.

as leukaemia,' Behçet's disease,' septic cavernous sinus thrombosis, ${ }^{1}$ subacute bacterial endocarditis, systemic lupus erythematosus, ${ }^{2}$ syphilis, ${ }^{3}$ and temporal arteritis. ${ }^{4}$ It is possible that in some of these cases the arterial and venous occlusions may not have occurred simultaneously.

Richards ${ }^{1}$ collected six cases of combined retinal vascular occlusion and described a characteristic clinical picture. His patients suffered a rapid reduction of the vision of one eye, often progressing to no light perception within a few days. They all experienced some retrobulbar pain. In each case the optic disc was swollen, with peripapillary retinal haemorrhages. The retina was pale, with a macular cherry red spot. A paucity of haemorrhages was noted in the peripheral retina, and Richards contrasted this with pure CRVO, in which retinal haemorrhages are usually more widespread. Fluorescein angiography showed lack of flow in the retinal vessels, with preservation of the choroidal circulation.

Our case differs from this description in several respects.

Timing of visual loss. In Richards's series, and in other reports, ${ }^{25}$ visual loss occurred over hours, and in some cases vision continued to deteriorate for several weeks. Our patient suffered complete loss of vision of the affected eye from the time of her blunt ocular injury. This may have been because the CRAO was more complete, or it may have been the result of an associated optic nerve injury.

Aetiology. The combination of CRAO and CRVO has previously been reported in eyes of patients with

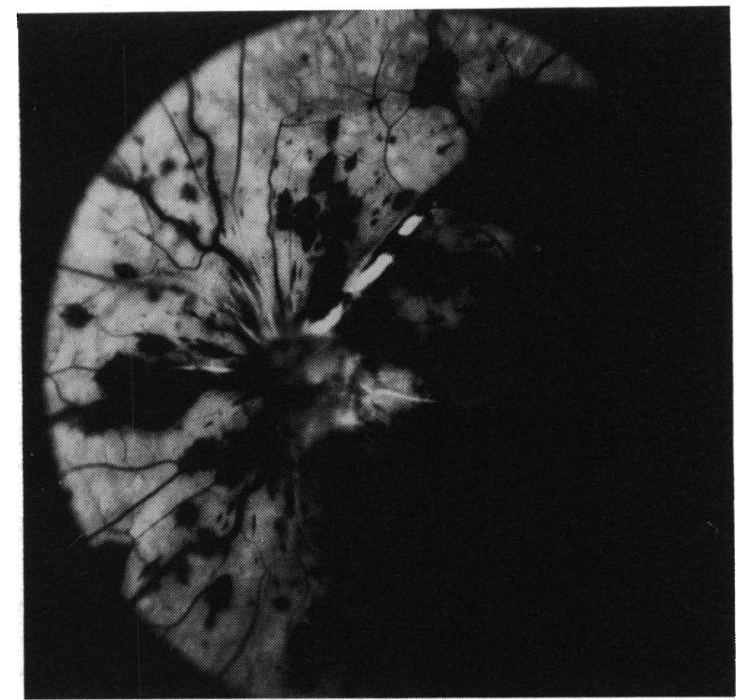

Fig. 2 Fluorescein angiogram frame of the left eye one day after injury (146 seconds after injection).

systemic disorders, particularly leukaemia. To our knowledge there is no previously recorded instance of a healthy patient suffering a combined central retinal vascular occlusion after blunt ocular trauma.

Fundus changes. All of Richards's cases had haemorrhages round the optic disc, with relative sparing of the peripheral retina. By contrast in our case retinal haemorrhages were initially few and scattered, but they increased over the ensuing 24 hours until florid retinal and preretinal haemorrhages involved in all regions of the fundus.

Other reports ${ }^{236}$ do not accord with Richards's fundus description. Bender ${ }^{6}$ described the case of a woman who suffered a combined retinal vascular occlusion 36 hours after a tooth extraction. On the morning that vision was lost, 'blurring of the right disc with profuse haemorrhages, exudate, and oedema throughout the retina' was observed.

During his investigation of the role of arterial ischaemia in retinal vein occlusion Hayreh reported the results of several series of experiments in which retinal arteries and/or veins of monkeys were occluded. He found that simultaneous occlusion of both central retinal artery and vein within the orbit by diathermy produced an initial picture of retinal pallor, with no blood flow within retinal vessels. ${ }^{7}$ Haemorrhages later developed in all three animals treated in this way. These were greatest round the optic disc or macula, and in one animal they were described as 'massive' and 'extensive'. This description bears similarities to the sequence of fundal changes in our clinical case.

In a later series of experiments Hayreh and 
colleagues repeated the occlusion of the central retinal vein and artery in the orbit of 11 monkeys. On this occasion they found "mild ischaemic odema ... associated with a few small venular haemorrhages (in one quarter)' though they noted that in one animal the retinal haemorrhages were far more numerous.

It is likely that combined CRAO and CRVO can produce widely differing patterns of clinical presentation and fundal signs depending upon such variables as (1) the relative severity of the arterial and venous occlusions; (2) the time course during which any arterial circulation is re-established; (3) the clinical condition producing the occlusions (for example, some of Richards's patients had lymphoma or leukaemia, and malignant infiltration may have contributed to the retinal pallor observed); (4) the patient's (or experimental animal's) age, since younger subjects may have a milder haemorrhagic response to retinal venous occlusion.

\section{MECHANISM}

The mechanism by which this combined retinal vascular occlusion was induced remains speculative. Haemorrhage within the optic nerve sheath can compress the nerve sufficiently to impair axonal transport. Jonasson and Cullen reported such a case caused by diving, in which the intrasheath haematoma also produced proptosis of the globe, and it mildly reduced vision." In our case the instantaneous loss of vision, the lack of proptosis, and the occurrence of a combined retinal vascular occlusion before the optic nerve head became swollen argue against such a mechanism.

There are a number of reported instances in which blunt ocular trauma has produced damage to the optic nerve, sometimes with degrees of nerve avulsion from the globe. ${ }^{11213}$ Severe ocular rotation has been cited as the cause of the optic nerve injury. ${ }^{1113}$ It is possible that extreme rotation induced by the finger jab may have produced damage to or haemorrhage in the retrolaminar part of the optic nerve in our patient. This in turn may have compressed or disrupted the central retinal vessels within the nerve.

We thank Mrs J Murray and Mrs S Mitchell for secretarial assistance, Mrs A Curric for photographs, and Dr G N Dutton for his permission to report this case and for his helpful comments.

\section{References}

1 Richards RD. Simultaneous occlusion of the central retinal artery and vein. Trans Am Ophthalmol Soc 1979; 77: 191-09.

2 Coppeto J, Lessel S. Retinopathy in systemic lupus erythematosus. Arch Ophthalmol 1977; 95: 984-7.

3 Smith JL. Acute blindness in early syphilis. Arch Ophthalmol 1973; 90: 256-8.

4 Cullen JF. Occult temporal arteritis. Br J Ophthalmol 1967; 51: 513-25.

5 Stowe CG III, Zakov ZN, Albert DM. Central retinal vascular occlusion associated with oral contraceptives. Am J Ophthalmol 1978: 86: 798-801.

6 Bender MB. Simultaneous closure of all the central retinal vessels. Am J Ophthalmol 1935; 18: 148-50.

7 Hayreh SS. Occlusion of the central retinal vessels. $\mathrm{Br} J$ Ophthalmol 1965; 49: 626-45.

8 Hayreh SS, van Heuven WAJ, Hayreh MS. Experimental retinal vascular occlusion. Arch Ophthalmol 1978; 96: 311-23.

9 Jonasson F, Cullen JF. Axonal transport injury caused by diving. Am J Ophthalmol 1982: 94: 813-5.

10 Juler FA. Rupture of the optic nerve at the nerve entrance with laceration of the inferior retinal vessels. Trans Ophthalmol Soc UK 1916; 36: 241-2

11 Stanton-Cook L. Injury simulating congenital anomaly. $\mathrm{Br} \mathrm{J}$ Ophthalmol 1953; 37: 188-9.

12 Treacher Collins E. Concussion injury with rupture extending across the optic disc. Trans Ophthalmol Soc UK 1914; 34: 190-2.

13 Spizziri LJ. Avulsion of optic nerve. Am J Ophthalmol 1964: 58: 1056-9.

Accepted for publication 13 November 1986. 